\title{
Magnetic Rotation in the $A=80$ Region: M1 Bands in Heavy Rb Isotopes
}

R. Schwengner, H. Schnare, S. Frauendorf, F. Dönau, L. Käubler, H. Prade, and E. Grosse

Institut für Kern- und Hadronenphysik, FZ Rossendorf, 01314 Dresden, Germany

\section{A. Jungclaus, K. P. Lieb, and C. Lingk}

II. Physikalisches Institut, Universität Göttingen, 37073 Göttingen, Germany

\section{S. Skoda and J. Eberth}

Institut für Kernphysik, Universität zu Köln, 50937 Köln, Germany

G. de Angelis, A. Gadea, E. Farnea, D. R. Napoli, and C. A. Ur

INFN, Laboratori Nazionali di Legnaro, 35020 Legnaro, Italy

and

\section{G. Lo Bianco}

INFN, Sezione di Milano, 20133

Milano, Italy

\author{
We have studied the isotopes ${ }^{82} \mathrm{Rb}_{45}$, \\ ${ }^{83} \mathrm{Rb}_{46}$, and ${ }^{84} \mathrm{Rb}_{47}$ to search for magnetic \\ rotation which is predicted in the tilted-axis \\ cranking model for a certain mass region \\ around $A=80$. Excited states in these nu- \\ clei were populated via the reaction \\ ${ }^{11} \mathrm{~B}+{ }^{76} \mathrm{Ge}$ with $E=50 \mathrm{MeV}$ at the XTU \\ tandem accelerator of the LNL Legnaro. \\ Based on a $\gamma$-coincidence experiment using \\ the spectrometer GASP we have found \\ magnetic dipole bands in each studied nu- \\ clide. The regular M1 bands observed in \\ the odd-odd nuclei ${ }^{82} \mathrm{Rb}$ and ${ }^{84} \mathrm{Rb}$ include \\ $B(\mathrm{M} 1) / B(\mathrm{E} 2)$ ratios decreasing smoothly \\ with increasing spin in a range of \\ $13^{-} \leq \mathrm{J}^{\pi} \leq 16^{-}$. These bands are inter- \\ preted in the tilted-axis cranking model on
}

the basis of four-quasiparticle configurations of the type $\pi(f p) \pi g_{9 / 2}^{2} \nu g_{9 / 2}$. This is the first evidence of magnetic rotation in the $A \approx 80$ region. In contrast, the M1 sequences in the odd-even nucleus ${ }^{83} \mathrm{Rb}$ are not regular, and the $B(\mathrm{M} 1) / B(\mathrm{E} 2)$ ratios show a pronounced staggering.

Key words: in-beam $\gamma$-spectroscopy; magnetic dipole bands; tilted-axis cranking model.

Accepted: July 22, 1999

Available online: http://www.nist.gov/jres

\section{Introduction}

In the tilted-axis cranking (TAC) model [1], which considers the rotation of the nucleus about axes tilted with respect to the principal axes, a new rotational mode referred to as magnetic rotation has been established. This mode is expected to appear in nuclei with small deformation, if multi-quasiparticle configurations are formed from high- $j$ proton particles and high- $j$ neutron holes or vice versa. The coupling of these configurations results in a large transverse magnetic moment. The rotating magnetic dipole gives rise to the emission of magnetic dipole (M1) radiation in contrast to the electric quadrupole (E2) radiation induced by the rotating deformed electric charge distribution in the case of conventional rotation. In the case of magnetic rotation the 
total spin is built up by the gradual alignment of the spins of the high- $j$ nucleons ("shears mechanism"). This concept has been applied for the first time to the M1 bands ("shears bands") discovered in nuclei around ${ }^{200} \mathrm{~Pb}$ [2]. The predicted decrease of the M1 transition strength with increasing spin caused by the gradual alignment of the individual spin vectors (closing of the shears) has recently been experimentally proven for the M1 bands in ${ }^{198,199} \mathrm{~Pb}$ [3]. Magnetic rotation is also predicted for other mass regions of the nuclear chart [4]. Indeed, it has recently been observed in ${ }^{105} \mathrm{Sn}[5],{ }^{110} \mathrm{Cd}[6]$, and ${ }^{139} \mathrm{Sm}$ [7].

Among the mass regions, where magnetic rotation is predicted to occur, there is also the region around $A=80$ [4]. There, the particle-like protons fill successively the $f p$ and the high- $j$ intruder $g_{9 / 2}$ levels while hole-like neutrons occupy the $g_{9 / 2}$ level. Indeed, sequences of intense M1 transitions starting at about $E \approx 3 \mathrm{MeV}$ have been found in several $\mathrm{Br}, \mathrm{Rb}$, and $\mathrm{Kr}$ isotopes (see, e.g., [8] and Refs. therein) but there is too little experimental information so far to prove the appearance of magnetic rotation. To search for experimental evidence of the predicted magnetic rotation in this region we have investigated the nuclides ${ }^{82} \mathrm{Rb}_{45},{ }^{83} \mathrm{Rb}_{46}$, and ${ }^{84} \mathrm{Rb}_{47}$.

\section{Experimental Results}

Excited states in ${ }^{82,83,84} \mathrm{Rb}$ were populated via the reaction ${ }^{11} \mathrm{~B}+{ }^{76} \mathrm{Ge}$ at $E=50 \mathrm{MeV}$ using the ${ }^{11} \mathrm{~B}$ beam of the XTU tandem accelerator of the LNL Legnaro. $\gamma$ rays were detected with the spectrometer GASP. A total of $1.5 \times 10^{8}$ three-fold coincidence events was recorded in a thin-target experiment. On the basis of this experiment we have found several new band structures with respect to previous work $[9,10]$. In particular, M1 bands have been found for the first time in each studied nuclide. Partial level schemes including these bands found in the present experiment are shown in Fig. 1. These level schemes result from $\gamma-\gamma$ and $\gamma-\gamma-\gamma$ coincidence relations and $\gamma$-ray intensities. Spin and parity assignments are based on $\gamma-\gamma$ directional correlations and deexcitation modes.

\section{Interpretation}

The M1 bands of negative parity observed in the odd-odd nuclei ${ }^{82} \mathrm{Rb}$ and ${ }^{84} \mathrm{Rb}$ are regular $\left(E_{\gamma} \propto \mathrm{J}\right)$. The $B(\mathrm{M} 1) / B(\mathrm{E} 2)$ ratios deduced from the intensities of transitions deexciting a certain state of the M1 band reach values up to $25\left(\mu_{N} / \mathrm{eb}\right)^{2}$ and decrease smoothly with increasing spin in a range of $13 \leq \mathrm{J} \leq 16$. This is an important characteristic of magnetic rotation. Thus, we have interpreted these bands in the framework of the TAC model [1]. In the calculations, the lowest-lying four-quasiparticle (4qp) configuration for $Z=37$ and $N=45,47$ turns out to be $\pi(f p) \pi g_{9 / 2}^{2} \nu g_{9 / 2}$, which has been adopted. The parameter $\kappa$ of the QQ interaction was adjusted such that in a calculation for the even-even neighbor ${ }^{82} \mathrm{Kr}$ the experimental $B\left(\mathrm{E} 2,2^{+} \rightarrow 0^{+}\right)$[11] value is reproduced and in the case of ${ }^{84} \mathrm{Rb}$ scaled according to $\kappa \propto A^{-5 / 3}$. An equilibrium deformation of $\epsilon_{2}=0.16$ was obtained for the adopted $4 q p$ configuration in both ${ }^{82,84} \mathrm{Rb}$. The nuclei turn out to be very soft with respect to $\gamma$ deformation with a tendency to positive values in ${ }^{82} \mathrm{Rb}$ but negative values in ${ }^{84} \mathrm{Rb}$. The values of $\gamma=20^{\circ}$ and $\gamma=-10^{\circ}$ are used for ${ }^{82} \mathrm{Rb}$ and ${ }^{84} \mathrm{Rb}$, respectively. The experimental and calculated $B(\mathrm{M} 1) / B$ (E2) ratios are compared in Fig. 2. The experimental values in ${ }^{82} \mathrm{Rb}$ are well reproduced in the calculations. This is also the case for ${ }^{84} \mathrm{Rb}$ up to $\hbar \omega \approx 0.7$ $\mathrm{MeV}$. The increase of the experimental values at higher frequency can not be described within the assumed $4 q p$ configuration. It is probably due to a change to a $6 q p$ configuration.

The $\mathrm{M} 1$ bands $\mathrm{C}$ and $\mathrm{D}$ in ${ }^{83} \mathrm{Rb}$ are irregular. Moreover, the experimental $B(\mathrm{M} 1) / B(\mathrm{E} 2)$ ratios of these bands shown in Fig. 2 display a pronounced staggering which is not compatible with regular shears bands. In contrast to the odd-odd nuclei, the breakup of a pair of neutrons is necessary in ${ }^{83} \mathrm{Rb}$ to generate $3 q p$ or $5 q p$ configurations of the shears type. This may drive the nuclear shape to very small quadrupole deformation, which is incapable of establishing a stable shears mechanism.

Summarizing, we have observed M1 bands in ${ }^{82} \mathrm{Rb}$, ${ }^{83} \mathrm{Rb}$, and ${ }^{84} \mathrm{Rb}$ for the first time. The $B(\mathrm{M} 1) / B(\mathrm{E} 2)$ ratios are of the order $10\left(\mu_{N} / \mathrm{eb}\right)^{2}$ to $20\left(\mu_{N} / \mathrm{eb}\right)^{2}$ and decrease with the angular momentum. This is characteristic for Magnetic Rotation. Thus, first evidence of the predicted existence of this new mode near $A=80$ has been provided. The M1 bands in the doubly odd nuclei ${ }^{82} \mathrm{Rb}$ and ${ }^{84} \mathrm{Rb}$ can be described in the TAC model on the basis of a $4 q p$ shears configuration. In contrast, the M1 bands in the odd-even nucleus ${ }^{83} \mathrm{Rb}$ are not regular. The difference may be caused by the breakup of a neutron pair driving the nucleus to substantially smaller deformation, which is incapable of sustaining the shears mechanism. 

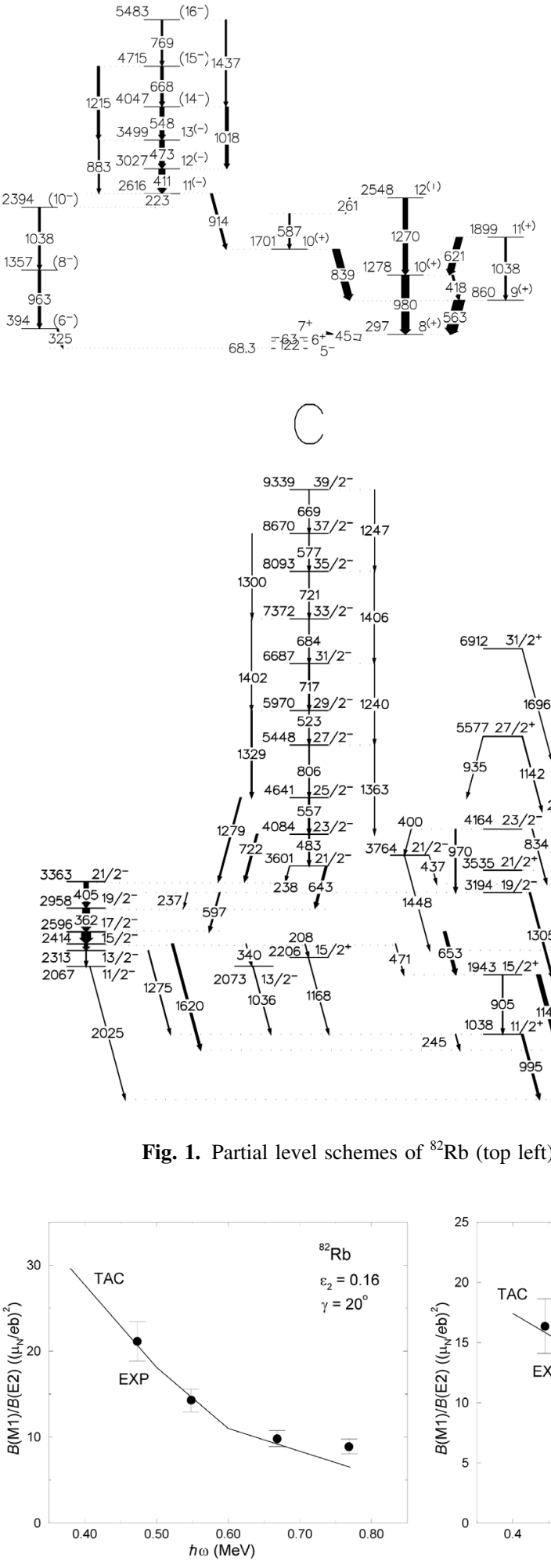

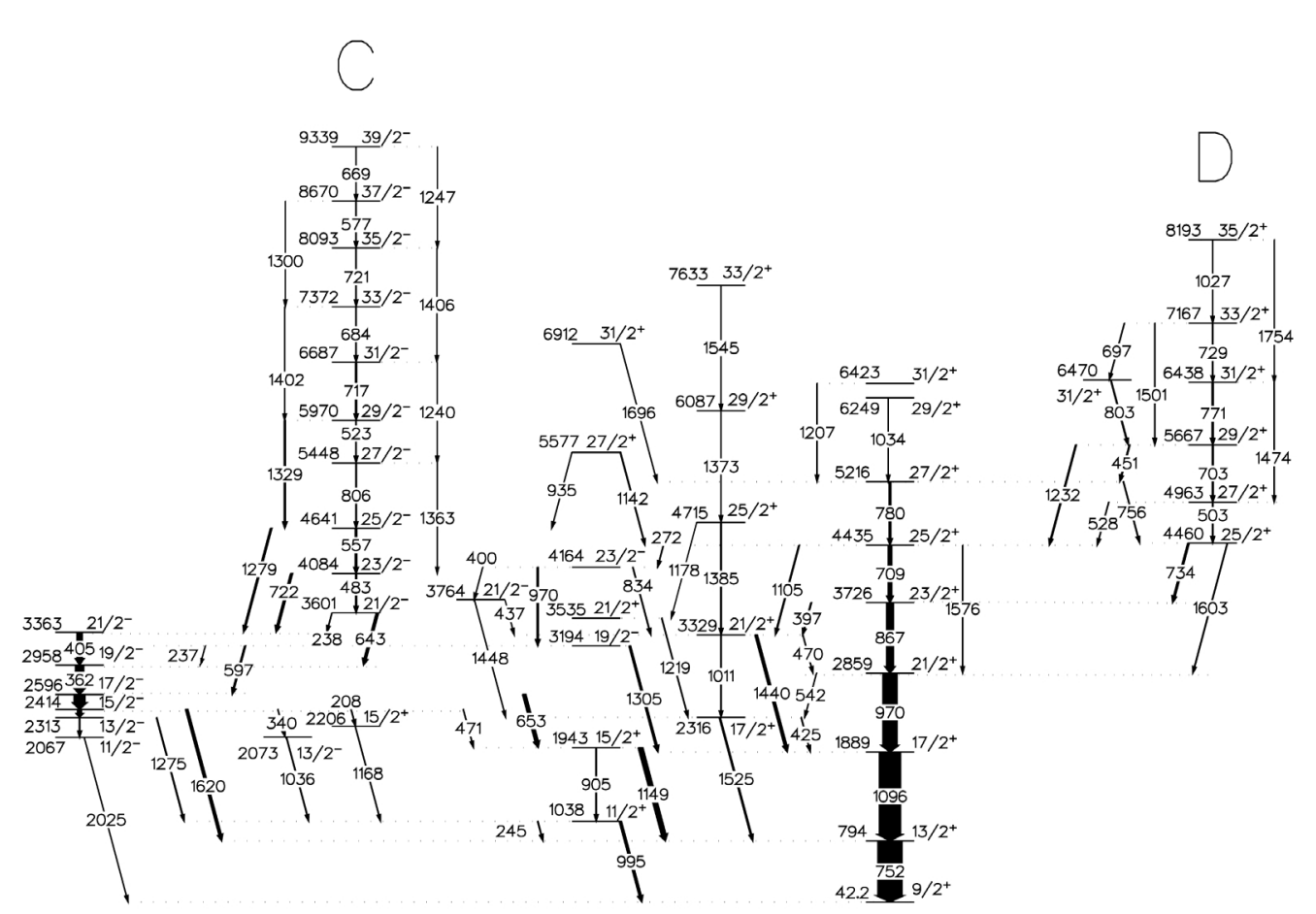

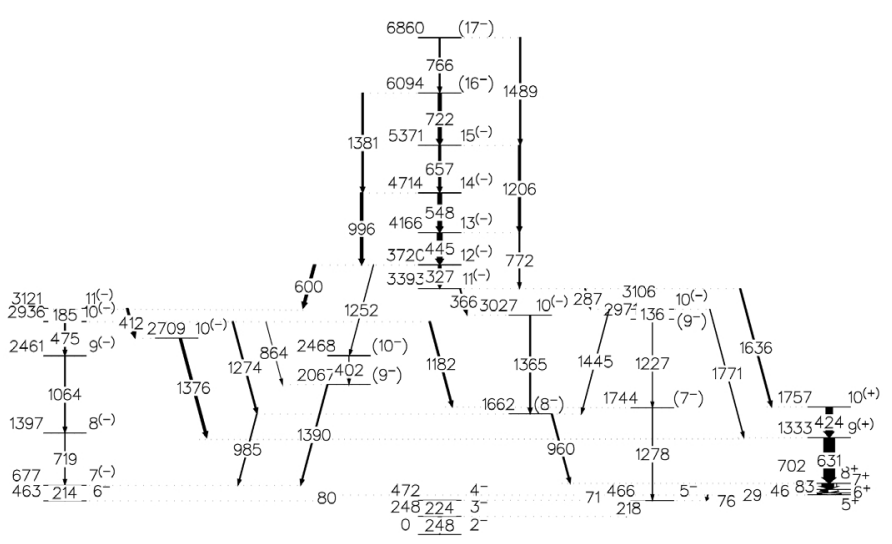

${ }^{82} \mathrm{Rb}$ (top left) and ${ }^{84} \mathrm{Rb}$ (top right) and ${ }^{83} \mathrm{Rb}$ (bottom) deduced from this work.
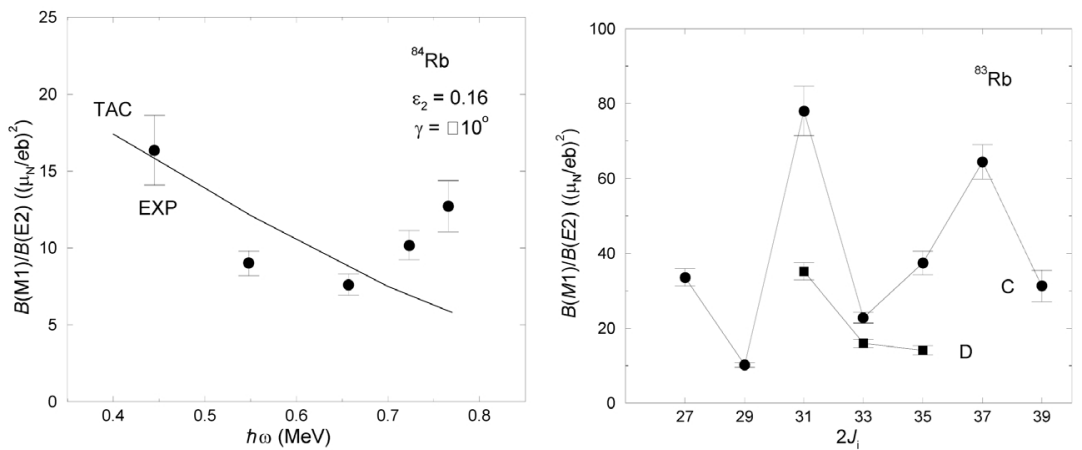

Fig. 2. Experimental and calculated $B(\mathrm{M} 1) / B(\mathrm{E} 2)$ ratios of the negative-parity $\mathrm{M} 1$ bands in ${ }^{82} \mathrm{Rb}$ (left panel) and ${ }^{84} \mathrm{Rb}$ (middle panel). Experimental $B(\mathrm{M} 1) / B(\mathrm{E} 2)$ ratios of the $\mathrm{M} 1$ bands $\mathrm{C}$ and $\mathrm{D}$ in ${ }^{83} \mathrm{Rb}$ (right panel). 


\section{Acknowledgment}

This work has been supported by the German Ministry of Education and Research (BMBF).

\section{References}

[1] S. Frauendorf, Nucl. Phys. A 557, 259c (1993).

[2] G. Baldsiefen, et al., Nucl. Phys. A 574, 521 (1994).

[3] R. M. Clark, et al., Phys. Rev. Lett. 78, 1868 (1997).

[4] S. Frauendorf, Z. Phys. A 358, 163 (1997).

[5] A. Gadea, et al., Phys. Rev. C 55, R1 (1997).

[6] S. Juutinen, et al., Nucl. Phys. A 573, 306 (1994).

[7] F. Brandolini, et al., Phys. Lett. B 388, 468 (1996).

[8] S. L. Tabor and J. Döring, Phys. Scr. T56, 175 (1995).

[9] J. Döring, et al., Z. Phys. A 338, 457 (1991), and Z. Phys. A 339, 425 (1991).

[10] W. Gast, et al., Phys. Rev. C 22, 469 (1980).

[11] P. Kemnitz, et al., Phys. Lett. B 125, 119 (1983).

About the authors: $R$. Schwengner is a senior researcher at the Forschungszentrum Rossendorf (FZR), $H$. Schnare is a post-doctoral scientist at FZR, S. Frauendorf is a Professor at Notre Dame and FZR, F. Dönau is head of the Nuclear Physics Division at FZR, L. Käubler is a senior researcher at FZR, H. Prade is head of the Radiation Physics Division at FZR, E. Grosse is Director of the Institute of Nuclear and Hadron Physics at FZR, A. Jungclaus is a post-doctoral scientist at the Uni Göttingen, K. P. Lieb is Professor a the Uni Göttingen, C. Lingk is a student at the Uni Göttingen, S. Skoda is a post-doctoral scientist at the Uni Köln, J. Eberth is a lecturer at the Uni Köln, G. de Angelis is a senior researcher at INFN-LN Legnaro, A. Gadea is a post-doctoral scientist at INFN-LN Legnaro, E. Farnea is a post-doctoral scientist at INFN-LN Legnaro, D. R. Napoli is a senior researcher at INFNLN Legnaro, C. A. Ur is a post-doctoral scientist at INFN-LN Legnaro, and G. Lo Bianco is a Professor at INFN-Sezione di Milano. 